\title{
The Nuclear Sigma Term in the Skyrme Model: Pion-Nucleus Interaction
}

\author{
A.Gammal ${ }^{1}$ and T.Frederico ${ }^{2}$ \\ ${ }^{1}$ FINPE, Instituto de Física, Universidade \\ de São Paulo, CP66318, 05315-970, São Paulo, Brazil \\ 2 Departamento de Física, Instituto Tecnológico da Aeronáutica, \\ Centro Técnico Aeroespacial, 12228-900 São José dos Campos, \\ São Paulo, Brazil
}

\begin{abstract}
The nuclear sigma term is calculated including the nuclear matrix element of the derivative of the NN interaction with respect to the quark mass, $m_{q} \frac{\partial V_{N N}}{\partial m_{q}}$. The NN potential is evaluated in the skyrmion-skyrmion picture within the quantized product ansatz. The contribution of the NN potential to the nuclear sigma term provides repulsion to the pion-nucleus interaction. The strength of the s-wave pion-nucleus optical potential is estimated including such contribution. The results are consistent with the analysis of the experimental data.
\end{abstract}

13.75.Cs,13.75.Gx,21.30.Cb,25.80.Dj 


\section{INTRODUCTION}

Nowadays Quantum Chromodynamics is accepted as the theory for strongly interacting particles. It was probed with success at high energies where perturbative expansion can be used. Unfortunately in the low energy regime the perturbative expansion is not valid and nonperturbative schemes such as lattice QCD have been implemented, but still with enormous computational efforts. The energy region in which quarks and gluons build the effective hadronic degrees of freedom is of interest for nuclear physics applications. Effective theories have been constructed considering only hadronic degrees of freedom and designed to work at low energies. In such models the main guides are the symmetries of the fundamental theory. A bridge can be made between the two descriptions when considering the QCD vacuum structure. The presence of hadronic matter affects the vacuum structure, which is reflected by the modification of the quark and gluon condensates [1]. The hadronic matter dig a hole in the local value of the condensate as expressed by the expectation value of the local composite operator $\bar{q} q$, where $q$ is the quark operator.

The difference between the volume integral of the quark condensate in the presence of hadronic matter and its vacuum value is proportional to the nuclear sigma term. It can be obtained by the application of the Hellmann-Feynman theorem, which allows to express the nuclear sigma term as $m_{q} \frac{\partial M_{A}}{\partial m_{q}}$ with $M_{A}$ being the nuclear mass [1]. We are limiting our discussion to the $\mathrm{SU}(2)$ flavor sector. The nucleon-nucleon interaction is responsible for the nuclear binding, so it is natural to question how the NN potential will be affected by variations of the quark masses, if a reliable calculation of the nuclear sigma term is desired [2].

However, to predict how the potential depends on the current quark masses of the fundamental theory, the connection of the effective hadronic model with QCD is necessary. The quark mass terms break explicitly the chiral symmetry in the QCD Lagrangian. In the SU(2) flavor sector of the theory, the up-down quark masses originate the pion mass, since the pion is considered the Goldstone boson arising from the spontaneous breaking of $\mathrm{SU}(2)_{\mathrm{L}} \times \mathrm{SU}(2)_{\mathrm{R}}$ chiral symmetry [3].

The Skyrme model allows to study the effect of the explicitly chiral symmetry breaking in a chiral invariant hadronic model. The quark mass comes through the pion mass term in the Skyrme Lagrangian. Such connection is made using the Gell-Mann, Oakes and Renner (GOR) relation [3],

$$
m_{\pi}^{2} f_{\pi}^{2}=-2 m_{q}\langle|\bar{q} q|\rangle_{v a c}
$$

where $m_{q}=\left(m_{u}+m_{d}\right) / 2, m_{u}$ and $m_{d}$ are the up and down current quark masses, respectively. The mass $m_{q}$ is quite small in the hadronic scale and the local value of the vacuum condensate is $\langle|\bar{q} q|\rangle_{v a c}:=\langle|\bar{u} u+\bar{d} d|\rangle_{v a c} / 2$.

The Skyrme Model [4,5] describes qualitatively the nucleon [6] and the nucleon-nucleon interaction [7 9]. The particular feature of the model is that the pion mass term in the Lagrangian contributes not only for the nucleon properties but also to the NN potential. We are supposing that the dependences of the pion weak decay constant $\left(f_{\pi}\right)$ and the Skyrme parameter $(e)$ on the up-down quark masses are small and they are not considered here.

The derivative of any hadronic quantity, $O$, with the up-down average quark mass in the Skyrme model is translated into a derivative in $m_{\pi}^{2}$ using the GOR relation, Eq.(1), such 
that

$$
m_{q} \frac{\partial O}{\partial m_{q}}=m_{\pi}^{2} \frac{\partial O}{\partial m_{\pi}^{2}}
$$

In this work, we obtain the derivative of the NN interaction with respect to the current quark mass by calculating the potential with the product ansatz approximation in the Skyrme model. We discuss how the derivative $m_{q} \frac{\partial V_{N N}}{\partial m_{q}}$ enters in the evaluation of the nuclear sigma term in the context of nonrelativistic quantum mechanics and its contribution

to the quark condensate. The expectation value of $m_{q} \frac{\partial V_{N N}}{\partial m_{q}}$ in the nuclear state contributes to the repulsive strength of the low-energy isoscalar pion-nucleus s-wave optical potential. These calculations can be tested through the comparison with the results of the pion-nucleus scattering and pionic atom data analysis [10 12. For completeness, we also calculate the nucleon sigma term, the derivatives of the pion-nucleon coupling constant $\left(g_{\pi N N}\right)$ and axial charge $\left(g_{A}\right)$.

In Section II, we discuss the nuclear sigma term in the context of nonrelativistic quantum mechanics, defining the part of the sigma term associated with the NN interaction. In Section III, we discuss the quark condensate at finite nuclear density with the inclusion of the new term. In Section IV, the Skyrme model is briefly discussed. In Section V, the results for the derivative of the NN potential, with respect to the quark mass, are presented together with the derivatives of the one-body observables. In Section VI, part of the main results of this work are presented, those correspond to the calculations of the nuclear sigma term for several nuclei. In Section VII, the contribution of the derivative of the NN potential to the pion s-wave optical potential is calculated and compared to the results of the analysis of experimental data. In Section VIII, we summarize our conclusions.

\section{THE NUCLEAR SIGMA TERM.}

The sigma term is a measure of the isoscalar content of the nuclear state $\left(\Psi_{A}\right)$, with $A$ nucleons, and thus it gives the strength of chiral symmetry breaking due to the $u$ and $d$ quark masses. It can be directly defined as the expectation value of the commutators of the axial charge $\left(Q^{5}\right)$ with the Hamiltonian [13, 14], given by

$$
\Sigma_{A}=\left\langle\Psi_{A}\left|\left[Q^{5},\left[Q^{5}, H\right]\right]\right| \Psi_{A}\right\rangle .
$$

In QCD framework it can be expressed as [14

$$
\Sigma_{A}=2 m_{q} \int d^{3} x\left(\left\langle\Psi_{A}|\bar{q}(\vec{x}) q(\vec{x})| \Psi_{A}\right\rangle-\langle 0|\bar{q}(\vec{x}) q(\vec{x})| 0\rangle\right)=m_{q} \frac{\partial M_{A}}{\partial m_{q}}
$$

it is proportional to the nonstrange quark condensate. The last equality in Eq. (四), follows from the Hellmann-Feynman theorem [1] at the level of the fundamental QCD theory, where the quark masses contribute to the Hamiltonian as $\delta H_{Q C D}=m_{u} \bar{u} u+m_{d} \bar{d} d$.

The nucleon sigma term is

$$
\Sigma_{N}=m_{q} \frac{\partial M_{N}}{\partial m_{q}}
$$


where we used (21) and $M_{N}$ is the nucleon mass. The experimental value extracted from pion-nucleon scattering is $\Sigma_{N} \sim 45 \mathrm{MeV}$ 15].

The nuclear sigma term has a contribution from the derivative of the nuclear binding energy $\left(E_{B}\right)$ with respect to the quark mass. Using that $M_{A}=Z M_{p}+(A-Z) M_{n}+E_{B}$, where $Z$ is the proton number, $M_{p}$ and $M_{n}$ are the proton and neutron masses, respectively, we have:

$$
\Sigma_{A}=A \Sigma_{N}+m_{q} \frac{\partial E_{B}}{\partial m_{q}}
$$

where the neutron and proton sigma terms were considered equal.

The effect of the nuclear binding in the sigma term can be calculated, for example, from the Schrödinger equation in the center of mass system. If the quark masses contribute effectively to $V_{N N}$, they also influence the binding energies in the Schrödinger equation, written as

$$
H\left|\Psi_{A}\right\rangle=\left[\sum_{i=1, A}-\frac{\hbar^{2}}{2 M_{N}} \nabla_{i}^{2}+\frac{1}{2} \sum_{i \neq j} V_{i j}\right]\left|\Psi_{A}\right\rangle=E_{B}\left|\Psi_{A}\right\rangle .
$$

Using the Hellman-Feynman theorem and considering that $M_{N}$ and $V_{i j}$ depend on $m_{q}$, the nuclear sigma term is given by

$$
\Sigma_{A}=A \Sigma_{N}+\Sigma_{A}^{K}+\Sigma_{A}^{V},
$$

where

$$
\Sigma_{A}^{V}=\left\langle\Psi_{A}\left|\frac{1}{2} \sum_{i \neq j} m_{q} \frac{\partial V_{i j}}{\partial m_{q}}\right| \Psi_{A}\right\rangle
$$

is the contribution of the NN potential to the nuclear sigma term, and

$$
\Sigma_{A}^{K}=-\frac{\Sigma_{N}}{M_{N}} \bar{E}_{K}
$$

is the term coming form the kinetic energy $\bar{E}_{K}$, which is the expectation value of the sum of the nucleon kinetic energies in the center of mass frame of the nucleus. The $A \Sigma_{N}$ term in Eq.(8) has been discussed in the context of nuclear matter in Ref. [1.2]. The last term in Eq. (8) can be rewritten using Eq.(2), as

$$
\frac{1}{2} \sum_{i \neq j} m_{q} \frac{\partial V_{i j}}{\partial m_{q}}=\frac{1}{2} \sum_{i \neq j} m_{\pi}^{2} \frac{\partial V_{i j}}{\partial m_{\pi}^{2}} .
$$

The above equality permits to calculate the derivative $m_{q} \frac{\partial V_{N N}}{\partial m_{q}}$ in the Skyrme model.

\section{THE QUARK CONDENSATE}

The nuclear sigma term is responsible for the change in the quark condensate at finite density [1.2]. The sigma term for nuclear matter at a finite density $(\rho)$ in a given volume $(\mathrm{Vol})$ is given by 


$$
\Sigma_{\rho}=2 m_{q} \operatorname{Vol}(\langle\rho|\bar{q}(\vec{x}) q(\vec{x})| \rho\rangle-\langle 0|\bar{q}(\vec{x}) q(\vec{x})| 0\rangle)=m_{q} \frac{\partial(\mathcal{E} V o l)}{\partial m_{q}}
$$

where $\mathcal{E}$ is the nuclear matter energy density given by $\mathcal{E}=M_{N} \rho+\frac{\bar{E}_{V}+\bar{E}_{K}}{A} \rho$, in the limit of $A \rightarrow \infty$ and $\bar{E}_{V}$ is the potential energy. Since $\rho=A / V o l$, we get from (12)

$$
2 m_{q}\left(\langle\bar{q} q\rangle_{\rho}-\langle\bar{q} q\rangle_{v a c}\right)=m_{q} \frac{\partial M_{N}}{\partial m_{q}} \rho+m_{q} \frac{\partial\left(\bar{E}_{V} / A+\bar{E}_{K} / A\right)}{\partial m_{q}} \rho .
$$

Using (8), the definitions $\Sigma_{A}^{V}(9), \Sigma_{A}^{K}(10)$ and the GOR relation, $2 m_{q}\langle\bar{q} q\rangle_{v a c}=-m_{\pi}^{2} f_{\pi}^{2}$, we have

$$
\frac{\langle\bar{q} q\rangle_{\rho}}{\langle\bar{q} q\rangle_{v a c}} \simeq 1-\frac{\Sigma_{N}+\left(\Sigma_{A}^{V}+\Sigma_{A}^{K}\right) / A}{m_{\pi}^{2} f_{\pi}^{2}} \rho+\ldots .
$$

If we neglect $\left(\Sigma_{A}^{V}+\Sigma_{A}^{K}\right)$ the expression above reduces to the leading order term found in Ref. [2]. Recent works by Li and Ko [16], Delfino et al [17] and Brockman and Weise [18], calculated the quark condensate in relativistic nuclear matter theories following the work of Cohen and collaborators [2]. They included in the evaluation of the nuclear sigma term the derivatives of the meson masses with respect to the quark mass, and in their context the quark condensate didn't deviate strongly from the leading order calculation at normal density. Brown and Rho [19] have studied the quark condensate in nuclear medium without the contribution of the NN potential to the nuclear sigma term. The values of the condensate at normal density, in all cases, are around the leading order result, $\langle\bar{q} q\rangle_{\rho_{N}} /\langle\bar{q} q\rangle_{v a c} \approx 0.70$.

In the following section we will calculate $\Sigma_{A}^{V}+\Sigma_{A}^{K}$ and $\Sigma_{A}$ using the Skyrme Model and the GOR relation. Subsequently we will calculate the contribution of $\Sigma_{A}^{V}$ to the pion-nucleus optical potential. In the summary, we will present an estimate to the quark condensate using (14), with $\Sigma_{A}^{V}$ obtained from the Skyrme model.

\section{THE SKYRME MODEL.}

The Skyrme Lagrangian [4] can be expressed as

$$
\mathcal{L}=\frac{f_{\pi}^{2}}{4} \operatorname{Tr}\left(\partial_{\mu} U \partial^{\mu} U^{\dagger}\right)+\frac{1}{32 e^{2}} \operatorname{Tr}\left[U^{\dagger} \partial_{\mu} U, U^{\dagger} \partial_{\nu} U\right]^{2}+\frac{1}{2} f_{\pi}^{2} m_{\pi}^{2}(\operatorname{Tr} U-2) .
$$

where $U=e^{i \vec{\tau} \cdot \vec{\pi}} ; \vec{\tau}$ are the Pauli isospin matrices. Assuming the hedgehog ansatz, $\vec{\pi}(\vec{r})=$ $\hat{r} F(r)$, and minimizing the energy we arrive at a differential equation for the profile function $(F)$

$$
\left(\frac{u^{2}}{4}+2 s^{2}\right) F^{\prime \prime}+\frac{1}{2} u F^{\prime}+F^{\prime 2} 2 s c-\frac{1}{2} s c-\frac{2 s^{3} c}{u^{2}}-\beta^{2} u^{2} \sin F=0,
$$

where $u=2 e f_{\pi} r, \beta=m_{\pi} /\left(2 e f_{\pi}\right), s=\sin F$ and $c=\cos F$. The solution with baryon number $\mathrm{B}=1$, can be achieved imposing $F(0)=\pi$ and $F(\infty)=0$.

We make use of the quantized version [7,8] of the product ansatz to get a qualitative idea of the consequences of finite quark masses in the NN potential. The product ansatz 
was originally introduced by Skyrme [5] as an attempted solution for the skyrmion-skyrmion interaction. It has the virtue of giving baryon number $\mathrm{B}=2$ for any separation distance and gives the one pion exchange potential for large distances. It provides an NN potential which can be decomposed in the form

$$
V_{N N}=V_{C}+\vec{\tau}_{1} \cdot \vec{\tau}_{2}\left[V_{S S}\left(\vec{\sigma}_{1} \cdot \vec{\sigma}_{2}\right)+V_{T} S_{12}\right]
$$

where $V_{C}$ is the isoscalar central potential, $V_{S S}$ is the spin-spin potential and $V_{T}$ is the tensor potential; $S_{12}=3\left(\vec{\sigma}_{1} . \hat{r}\right)\left(\vec{\sigma}_{2} \cdot \hat{r}\right)-\vec{\sigma}_{1} \cdot \vec{\sigma}_{2} . ; \vec{\sigma}$ are the Pauli spin matrices.

Exact numerical solutions for the skyrmion-skyrmion interaction were obtained in the work of Walhout and Wambach [9]. In that work [9], they compared the exact numerical calculations to the product ansatz result and to the Paris potential. Although the gross feature of the central component is given by the product ansatz, it misses the attraction which is present in the exact calculation and Paris potential. The product ansatz is roughly reliable for $r \geq 1.5 \mathrm{fm}$ in the tensor and spin-spin channels.

Although the product ansatz in the NN central potential is only approximated when compared to exact numerical calculations [9], we believe that is possible to use it in the calculation of the derivative. According to Jackson et al [7], the difficulties in reproducing the NN interaction from the product ansatz come from the fact that the potential is a difference of two large quantities of magnitudes two orders higher than the potential itself, and so little deformation in the skyrmions can originate drastic effects. This shall not occur in the derivative of the potential with respect to the quark mass, since the final result is of the same order of magnitude of the individual nucleon contributions. So, deformations that could occur in the skyrmions as they are close, would be in this case only corrections that could not change the magnitude of the result.

\section{DERIVATIVES OF THE NUCLEON OBSERVABLES AND NN POTENTIAL}

We begin by showing the results of the derivatives of the one-body observables with respect to the quark mass. We can calculate several nucleon observables [6], such as the nucleon mass, the pion-nucleon coupling $\left(g_{\pi N N}\right)$ and the axial coupling $\left(g_{A}\right)$, from the Skyrme Model in the semi-classical quantization approach. Thus, by changing the pion mass we can easily calculate $m_{\pi}^{2} \frac{\partial M_{N}}{\partial m_{\pi}^{2}}, m_{\pi}^{2} \frac{\partial g_{\pi N N}}{\partial m_{\pi}^{2}}$ and $m_{\pi}^{2} \frac{\partial g_{A}}{\partial m_{\pi}^{2}}$. These calculations provide the determination of the sigma term, $m_{q} \frac{\partial g_{\pi N N}}{\partial m_{q}}$ and $m_{q} \frac{\partial g_{A}}{\partial m_{q}}$, respectively. The results of the derivatives of the observables are presented in Table I, and for completeness, the value of the observables themselves are also shown.

The calculations were performed with two sets of parameters, $f_{\pi}=54 \mathrm{MeV}, e=4.84$ [6] and $f_{\pi}=93 \mathrm{MeV}, e=4.0$ [20] with $m_{\pi}=138 \mathrm{MeV}$. In Table I, the values of the nucleon sigma term, were included. It has been calculated previously in Ref. [6]. They obtained 38 $\mathrm{MeV}$ and $49 \mathrm{MeV}$, for the set $f_{\pi}=54 \mathrm{MeV}$ and $e=4.84$, using different methods without recurring to the Hellmann-Feynman theorem. These values are comparable to our result of $52.2 \mathrm{MeV}$. The experimental value of about $45 \mathrm{MeV}$ [15], is somewhat consistent with our value of $59.6 \mathrm{MeV}$ for $f_{\pi}=93 \mathrm{MeV}$ and $e=4.0$ (see Table I). We will restrict our evaluation of the nuclear sigma term to this set of parameters, since $f_{\pi}$ is taken from the experimental value. 
The coupling constant $g_{\pi N N}$ has very different derivatives for the two sets of parameters, while the derivatives of $g_{A}$ are similar, as shown in Table I. However, in the case of the $g_{\pi N N}$, the derivatives $m_{q} \frac{\partial g_{\pi N N}}{\partial m_{q}}$, the nucleon sigma term and $m_{q} \frac{\partial g_{A}}{\partial m_{q}}$ are consistent with the Goldberger-Treiman relation [14].

The derivative $m_{q} \frac{\partial V_{N N}}{\partial m_{q}}$ is evaluated numerically in the Skyrme model using the quantized product ansatz with the parameter set $f_{\pi}=93 \mathrm{MeV}$ and $e=4.0$. We calculated the NN interaction in skyrmion-skyrmion picture and recalculated it for a small change in the pion mass. Thus, we obtained numerically the quantities $m_{q} \frac{\partial V_{C}}{\partial m_{q}}, m_{q} \frac{\partial V_{T}}{\partial m_{q}}$ and $m_{q} \frac{\partial V_{S S}}{\partial m_{q}}$. They are shown in Figs. 1, 2 and 3, respectively. The spin-spin and tensor potential derivatives are compared to those extracted from the one pion exchange potential (OPEP), presented in the appendix, where $g_{A}$ and $m_{q} \frac{\partial g_{A}}{\partial m_{q}}$ were taken from Table I.

The results for $m_{q} \frac{\partial V_{C}}{\partial m_{q}}$ as a function of the relative distance of the skyrmion centers, are shown in Fig. 1. The calculations show minimum around $1 \mathrm{fm}$, with values about -20 $\mathrm{MeV}$, while at short distance they present repulsion. The difference $V_{C}^{\left(m_{\pi}=138\right)}-V_{C}^{\left(m_{\pi}=0\right)}$ is comparable to $m_{q} \frac{\partial V_{C}}{\partial m_{q}}$, as shown in the figure.

In Fig.2, the results for $m_{q} \frac{\partial V_{T}}{\partial m_{q}}$ are shown. The derivative $m_{q} \frac{\partial V_{T}^{O P E P}}{\partial m_{q}}$ is compared with the corresponding one for the skyrmion-skyrmion interaction. They agree for separations of the nucleons above $3 \mathrm{fm}$. This is expected since the OPEP tail is present in the skyrmionskyrmion interaction. The derivative found with OPEP is negative at short-distances, while the product ansatz results are positive. The shapes of the curves for the difference $V_{T}^{\left(m_{\pi}=138\right)}-V_{T}^{\left(m_{\pi}=0\right)}$ and $m_{q} \frac{\partial V_{T}}{\partial m_{q}}$ are qualitatively similar, although the tail presents a noticeable difference. We have checked that at distances above $3 \mathrm{fm}$, the difference in the potentials with finite and zero pion masses is given by the results from OPEP, since the tail is dominated by $m_{\pi}$ being zero or finite.

The derivative of the spin-spin component of the skyrmion-skyrmion interaction, $m_{q} \frac{\partial V_{S S}}{\partial m_{q}}$, is shown in Fig. 3. The derivative of the OPEP spin-spin component for distances above 3 $\mathrm{fm}$ agrees with the present calculation. As expected for distances below $2 \mathrm{fm}$, the derivatives of the skyrmion-skyrmion potential and OPEP become quite different. The shape of the

difference $V_{S S}^{\left(m_{\pi}=138\right)}-V_{S S}^{\left(m_{\pi}=0\right)}$ is similar to that of $m_{q} \frac{\partial V_{S S}}{\partial m_{q}}$. However at distances above 3 $\mathrm{fm}$, the difference in the potentials with finite and zero pion masses is essentially dominated by the results from OPEP, which explains the origin in the differences found.

\section{RESULTS FOR THE NUCLEAR SIGMA TERM}

One way to extract the nuclear sigma term is from the low energy pion-nucleus scattering analysis. In fact, in a recent work of Brown and Rho [19] it is suggested the possibility of obtaining information about the quark condensate from pionic atoms.

The NN potential extracted from the product ansatz is not realistic in the sense that it does not reproduce the central attraction in the mid-range. This cannot be ignored since it means that there is no wave function for the bound state in this potential. Then we decided to give up consistency and from the derivative of the calculated potential, we can estimate its contribution to the nuclear sigma term using charge densities from the electron elastic scattering [21]. 
In the particular case of the deuteron, we calculated

$$
\Sigma_{2_{\mathrm{H}}}^{V}=\left\langle\psi_{2_{\mathrm{H}}}\left|m_{q} \frac{\partial V}{\partial m_{q}}\right| \psi_{2_{\mathrm{H}}}\right\rangle
$$

using the normalized wave function [22].

$$
\psi_{2_{\mathrm{H}}}=\psi_{J=1, M}=(4 \pi)^{-1 / 2}\left\{\frac{u(r)}{r}+\frac{w(r)}{r} \frac{1}{\sqrt{8}} S_{12}(\hat{r})\right\} \chi_{1 M} .
$$

where $u$ and $w$ are the s-wave $\left({ }^{3} \mathrm{~S}_{1}\right)$ and $\mathrm{d}$-wave $\left({ }^{3} \mathrm{D}_{1}\right)$ from the deuteron wave function obtained with the Reid potential [23]. The normalized wave function is such that $\int_{0}^{\infty} d r\left(u^{2}+w^{2}\right)=1$. The spin component $\chi_{1 M} \equiv|1 M\rangle$ is such that $\chi_{1 M} \chi_{1 M}^{\dagger}=1$ and $r=\left|r_{1}-r_{2}\right|$ is the relative distance between the nucleons.

The Skyrme model offers naturally forces of two, three and more bodies. Nevertheless, it is still a good approximation to neglect them all in the nuclear sigma term except the contributions from the two-body potential. Assuming a modified Hartree approximation such that it has the correct number of pairs, we have

$$
\Sigma_{A}^{V}=\frac{A(A-1)}{2} \int d^{3} \mathbf{r}_{1} \rho\left(\mathbf{r}_{1}\right) \int d^{3} \mathbf{r}_{2} \rho\left(\mathbf{r}_{2}\right) m_{q} \frac{\partial V\left(\left|\mathbf{r}_{1}-\mathbf{r}_{2}\right|\right)}{\partial m_{q}},
$$

where $\int d^{3} \mathbf{r} \rho(\mathbf{r})=1$ and $A$ is the mass number.

For isoscalar nuclei heavier than the deuteron we neglected the spin-spin and tensor components of the derivative of the potential, considering that they are more than one order of magnitude smaller than the central part and the isospin is averaged in the matrix element (which yields zero in the Hartree approximation). Then only the derivative of the central potential enters in the estimate of $\Sigma_{A}^{V}$.

We present in Table II the results of $\Sigma_{A}^{V}$ using (20) and the densities obtained from elastic electron scattering [21]. In this first approach, we assumed the same proton and neutron density distributions. The deuteron results were included in Table II. In the case of the deuteron the contribution from the $\mathrm{NN}$ interaction is $-3.60 \mathrm{MeV}$. We expected that we could roughly count the number of NN pairs for light nuclei in $\Sigma_{A}^{V}$. For instance, in ${ }^{4} \mathrm{He}$ we can count 6 pairs, then we should have $6 \times(-3.60) \mathrm{MeV}=-21 \mathrm{MeV}$ that is a lower value (in modulus) than the calculated $-34.5 \mathrm{MeV}$. A possible explanation is that the deuteron is "big" and its NN interaction is on an average weaker than in ${ }^{4} \mathrm{He}$. Triton has 3 pairs and we estimate the contribution of the potential to the nuclear sigma term as being between $3 \times \Sigma_{2 \mathrm{H}}^{V}=10.8 \mathrm{MeV}$ and $3 \times \sum_{4 \mathrm{He}}^{V} / 6=17.2 \mathrm{MeV}$. Averaging we find a value of $14 \mathrm{MeV}$ for triton. The same should work for ${ }^{3} \mathrm{He}$. For larger values of $A, \Sigma_{A}^{V}$ remains always smaller than the number of pairs $\times \sum_{{ }_{2 \mathrm{H}}}^{V}$ or the number of pairs $\times \sum_{{ }_{4} \mathrm{He}}^{V} / 6$, because the NN interaction has a range around $1 \mathrm{fm}$. Also we observe that $\Sigma_{A}^{V} / A$ is always growing with $A$ except for the ${ }^{197} \mathrm{Au}$ and ${ }^{208} \mathrm{~Pb}$. This can be explained by considering that lead have lower density distribution at mid-range than gold, as we checked.

In Table II, we also included the kinetic energy contribution to the sigma term. The kinetic energies from the deuteron, ${ }^{3} \mathrm{He}$ and ${ }^{4} \mathrm{He}$ were extracted from the work of Schiavilla et al [24]. In the heavier nuclei we took the average kinetic energy of nonrelativistic nucleons in nuclear matter, given by $\bar{E}_{K} / A=\frac{3}{10}(2) \varepsilon_{f}$. At $\rho=0.17 \mathrm{fm}^{-3}, k_{f}=1.36 \mathrm{fm}^{-1}, \varepsilon_{f}=38 \mathrm{MeV}$ 
and we arrive at $\bar{E}_{K} / A=22.8 \mathrm{MeV}$. The values of $\Sigma_{A}^{V+K} \equiv \Sigma_{A}^{V}+\Sigma_{A}^{K}$ are also presented and they will be used in the evaluation of pion-nucleus optical potential.

We noticed that the calculation of the sigma term from the NN potential using the Gell-Mann, Oakes and Renner relation(GOR) and taking the derivative with respect to the pion mass, produced very different results from those calculated considering only the symmetry breaking term (according to Adkins and Nappi [6]). This occurs because there is an enormous contribution from the fourth order term. We also observed that it is in the mid-range $(1 \mathrm{fm}-2 \mathrm{fm})$ that the potential NN gives the biggest contribution to the sigma term.

\section{THE NUCLEAR SIGMA TERM IN THE PION-NUCLEUS S-WAVE POTENTIAL}

Considering that in the last section we got an estimate of the contribution of the NN potential to the nuclear sigma term, we can also use it in the calculation of the s-wave pion-nucleus potential. The interaction Lagrangian between the pion and the nucleus which is originated by the nuclear $\operatorname{sigma} \operatorname{term}\left(\Sigma_{A}\right)$ is given by

$$
\mathcal{L}_{\text {int }}^{\Sigma}=\frac{m_{q}}{2 f_{\pi}^{2}}\left\langle\psi_{A}|: \bar{q}(x) q(x):| \psi_{A}\right\rangle \pi^{2}
$$

We can approximate

$$
m_{q}\left\langle\psi_{A}|: \bar{q}(x) q(x):| \psi_{A}\right\rangle \simeq \frac{\Sigma_{A}}{A} \rho(\mathbf{r})
$$

with $\int d^{3} \mathbf{r} \rho(\mathbf{r})=A$. The nuclear sigma term is separated in its contributions of individual nucleons, potential and kinetic energy; $\Sigma_{A}=A \Sigma_{N}+\Sigma_{A}^{V}+\Sigma_{A}^{K}$ (see Eq. 8).

The component of the interaction Lagrangian which originates the s-wave potential $\left(U^{l=0}(\mathbf{r})\right)$ can be written as

$$
\mathcal{L}_{\mathrm{int}}^{l=0}=-\frac{\Pi^{l=0}(\mathbf{r})}{2} \pi^{2}
$$

where the s-wave self energy is given by

$$
\Pi^{l=0}(\mathbf{r})=-4 \pi\left(1+m_{\pi} / M_{N}\right)\left[b_{0_{\mathrm{eff}}}^{\text {free }}+\frac{\Sigma_{A}^{V+K}}{4 \pi f_{\pi}^{2} A}\left(1+\frac{m_{\pi}}{M_{N}}\right)^{-1}\right] \rho(\mathbf{r}) .
$$

We have separated $\Sigma_{A}^{V+K}$ and $b_{0_{\text {eff }}}^{\text {ree }}$ contributions in the self energy $\Pi^{l=0}(\mathbf{r})$, Eq. (24). This last one should contain all the mechanisms of the pion-nucleus interaction not included in $\Sigma_{A}^{V+K}$. The normalization was chosen such that $b_{0_{\mathrm{eff}}}^{\text {free }}$ has the same normalization as the $\pi N$ scattering length.

Introducing the self energy, $\Pi^{l=0}(\mathbf{r})$, in Klein-Gordon equation for the pion [22,25], using $\omega^{2}=k^{2}+m_{\pi}^{2}$ and dividing by $2 m_{\pi}$, we get

$$
\left[-\frac{\nabla^{2}}{2 m_{\pi}}+\frac{2 \omega V_{\text {Coul }}}{2 m_{\pi}}-\frac{V_{\text {Coul }}^{2}}{2 m_{\pi}}+\frac{\Pi^{l=0}(\mathbf{r})}{2 m_{\pi}}\right] \varphi_{a}(\mathbf{r})=\frac{k^{2}}{2 m_{\pi}} \varphi_{a}(\mathbf{r}) .
$$


In this way we have obtained the pion Schrödinger equation, where we identify the potential

$$
U^{l=0}(\mathbf{r})=\frac{1}{2 m_{\pi}} \Pi^{l=0}(\mathbf{r})
$$

The part of the s-wave optical potential from the term $\Sigma_{A}^{V+K}$ is given by

$$
U_{\Sigma_{A}^{V+K}}^{l=0}(\mathbf{r})=-\frac{\Sigma_{A}^{V+K}}{2 m_{\pi} f_{\pi}^{2} A} \rho(\mathbf{r})
$$

where Eq.s (24) and (26) were used. Then, if $\Sigma_{A}^{V+K}$ is positive, it will produce an attractive contribution to the optical potential and repulsive otherwise.

The scattering amplitude in the long-wave length limit and in the Born approximation is given by

$$
f(\mathbf{k})=-\frac{\mu_{\pi}}{2 \pi} \int d^{3} \mathbf{r} e^{i \mathbf{k} \cdot \mathbf{r}} U^{l=0}(\mathbf{r})
$$

The nucleus recoil is considered in Eq.(28) by the reduced mass factor $\mu_{\pi}=m_{\pi} M_{A} /\left(m_{\pi}+\right.$ $M_{A}$ ), that corresponds to the addition of the nonrelativistic kinetic energy of the target in the pion Schrödinger equation (25). The pion-nucleus scattering length in Born approximation is given by $a=f(0)$.

Although we have discussed the Born approximation in general, it only will be used for the deuteron, ${ }^{3} \mathrm{He}$ and ${ }^{4} \mathrm{He}$, when discussing experimental data. For heavier nuclei we will compare the calculations to the Seki-Masutani parameter $b_{0}^{\mathrm{SM}}$ 11] extracted from experimental data analysis and defined as the coefficient of the $\rho(\mathbf{r})$ term in the optical potential,

$$
2 m_{\pi} U^{l=0}(\mathbf{r})=-4 \pi\left(1+m_{\pi} / M_{N}\right) b_{0}^{\mathrm{SM}} \rho(\mathbf{r}) .
$$

Comparing the equations $(24),(26)$ and (29), we get

$$
b_{0}^{\mathrm{SM}}=b_{0_{\mathrm{eff}}}^{\text {free }}+b_{0}^{V+K},
$$

where,

$$
b_{0}^{V+K}=\frac{\Sigma_{A}^{V+K}}{4 \pi f_{\pi}^{2} A}\left(1+\frac{m_{\pi}}{M_{N}}\right)^{-1}
$$

which is the contribution of the nuclear sigma term from the NN potential plus the kinetic energy to $b_{0}^{\mathrm{SM}}$.

The isoscalar scattering length $b_{0_{\mathrm{eff}}}^{\text {free }}$ was obtained using the experimental value of the $\pi d$ scattering length $\left(\operatorname{Re} a_{\pi d}=-0.0264(11) m_{\pi}^{-1}\right)$ [26],

$$
b_{0_{\mathrm{eff}}}^{\text {free }}=\left(1+\frac{m_{\pi}}{M_{N}}\right)^{-1}\left[\left(1+\frac{m_{\pi}}{M_{d}}\right) \frac{\operatorname{Re} a_{\pi d}}{2}-\frac{\Sigma_{d}^{V+K}}{8 \pi f_{\pi}^{2}}\right],
$$

with $m_{\pi}=138 \mathrm{MeV}, M_{N}=938 \mathrm{MeV}, M_{d}$ is the deuteron mass, $f_{\pi}=93 \mathrm{MeV}$. We used $\Sigma_{2 \mathrm{H}}^{V+K}=-4.46 \mathrm{MeV}$, extracted from Table II, for $f_{\pi}=93 \mathrm{MeV}$ and $e=4.0$. These parameters adjust the $\pi N$ p-wave scattering 20]. Substituting these values in (32) we get 


$$
b_{0_{\mathrm{eff}}}^{\text {free }}=-0.0099\left(m_{\pi}^{-1}\right)
$$

This value is consistent with $-0.0077(11) m_{\pi}^{-1}$ got from Sigg et al [27] in the isospin symmetry hypothesis and also with the value $-0.0083(38) m_{\pi}^{-1}$ from Koch [28.

It has been noted that the scattering length for bound nuclei is shifted from the scattering length predicted by the theory for free nucleons?. This suggests that the cause for this shift is precisely the nuclear sigma term and kinetic energy contribution. The value of the isoscalar $\pi N$ scattering length parameter $b_{0}$ is controversial and is not our purpose to calculate it here. Then, we obtained the effective isoscalar $b_{0_{\mathrm{eff}}}^{\text {free }}$ originated from experimental pion-deuteron scattering length that contains the single and double scattering processes not included in $\Sigma_{2}^{V+K}$.

We constructed Table III using the results of the potential and kinetic energy contributions to the sigma term in Eq.(30) . The experimental results were extracted from pionic atoms and pion-nucleus scattering analysis [10,11].

In the particular case of ${ }^{3} \mathrm{He}, b_{0_{\mathrm{eff}}}^{(\exp }$ was evaluated subtracting the isovector contribution, i.e., considering only single scattering,

$$
b_{0_{\mathrm{eff}} \pi^{-} A}^{(\exp )}=\frac{1}{A}\left[\left(1+\frac{m_{\pi}}{M_{N}}\right)^{-1}\left(1+\frac{m_{\pi}}{A M_{N}}\right) \operatorname{Re} a_{\pi^{-} A}-(N-Z) b_{1}\right]
$$

and using the experimental values $\operatorname{Re} a_{\pi^{-}{ }^{3} \mathrm{He}}=0.056(6)$ 22 and $b_{1}=-0.0962(7)$ extracted from [27] we got $b_{0_{\mathrm{eff}}}^{(\exp )}\left({ }^{3} \mathrm{He}\right) \approx-0.015$. The experimental result for ${ }^{12} \mathrm{C}$ was obtained by the Seki-Masutani analysis and also for ${ }^{16} \mathrm{O}$ extrapolated from $\pi^{-}$scattering data at $29 \mathrm{MeV}$ and $50 \mathrm{MeV}$ [10. The experimental result for ${ }^{40} \mathrm{Ca}$ was found in the Masutani-Seki analysis [11] extrapolated from $\pi^{-}$scattering data at $25 \mathrm{MeV}$ to zero energy. In the cited work [10], it is also found that the pion interacts with the nucleus at an effective density $\rho_{e}=\rho_{N} / 2$. The empirical value of $-0.0304 m_{\pi}^{-1}$ was obtained by Salcedo et al [12 averaging values obtained from the analysis of pionic atom and pion-nucleus scattering data.

Our evaluation of the nuclear sigma term from the NN potential generates a repulsion in the pion- ${ }^{12} \mathrm{C}$ optical potential in the central region which is approximately $U_{\Sigma}^{V+K}(\mathbf{r})=$ $-\frac{\Sigma_{A}^{V+K}}{2 m_{\pi} f_{\pi}^{2} A} \rho(\mathbf{r}) \approx 8 \mathrm{MeV}$ that is roughly of the same order necessary to fit the pionic atoms ( $\approx 15$ to $30 \mathrm{MeV}$ ) and is "weakly dependent of $A$ " [30].

\section{CONCLUSIONS}

We have calculated the derivative of the skyrmion-skyrmion interaction with respect to the quark mass, $m_{q} \frac{\partial V_{N N}}{\partial m_{q}}$, in the quantized product ansatz. We have related it to $m_{\pi}^{2} \frac{\partial V_{N N}}{\partial m_{\pi}^{2}}$

\footnotetext{
${ }^{1}$ ¿From Kluge [30]: "The conclusion is that this repulsion cannot be obtained by an iteration of $\pi N$ interaction, but rather represents an independent feature of the interaction of pions with bulk nuclear matter. An attempt to explain this as a binding effect of the nucleons has been undertaken in the framework of relativistic mean field theory of the nucleus (Birbair et al 1983 J.Phys.G: Nucl.Phys 9 1473; Goudsmit et al 1989 Preprint ETHZ-IMP RP-90-03 ETH Zürich)"
} 
using the Gell-Mann, Oakes and Renner relation, which made the calculation possible. We compared it to the OPEP calculation, and they agreed for distances above $3 \mathrm{fm}$. The matrix element of above derivative of $V_{N N}$ in the nuclear state is part of the nuclear sigma term. The magnitudes of the quark mass contribution to the nuclear potential were found appreciable. We calculated the contribution to the nuclear sigma term arising from the nuclear interaction, assuming that the nuclear density in finite nuclei is equal to the experimental charge density conveniently normalized.

The contribution from the NN potential to the sigma term was incorporated in the effective isoscalar scattering length of the pion-nucleus optical potential. There was a remarkable agreement with the empirical values extracted from the analysis of pionic atoms and scattering experimental data. We showed that it is possible to explain the repulsion in the isoscalar channel of the pion-nucleus optical potential, if we take into account in the nuclear sigma term the contribution of the NN potential.

In nuclear matter, we should include the contribution of the NN potential $\left(\Sigma_{A}^{V}\right)$ in the nuclear sigma term in the form $\Sigma_{A}=A \Sigma_{N}+\Sigma_{A}^{V}+\Sigma_{A}^{K}$. This correction alters the value of the condensate $\langle\bar{q} q\rangle_{\rho} /\langle\bar{q} q\rangle_{v a c}=1-\rho \Sigma_{A} / A m_{\pi}^{2} f_{\pi}^{2}$, and as $\Sigma_{A}^{V}$ is negative there is a tendency to a drastic reduction of $\Sigma_{A}$. Then the condensate at normal nuclear density is about $\langle\bar{q} q\rangle_{\rho_{N}} /\langle\bar{q} q\rangle_{v a c} \approx 0.95$, and by this way it does not decrease as much as it was calculated before. More definitive conclusions about the quark mass dependence of the NN potential, should be pursued in exact evaluations of the skyrmion-skyrmion interaction, that is beyond the present work.

\section{Acknowledgments}

AG would like to thank M.R. Robilotta for the introduction to the Skyrme model. We also thank M.R. Robilotta and J.M.Eisenberg for valuable discussions on this subject. We express our gratitute to Vera B. Canteiro for reading the manuscript. This work was supported by FAPESP and CNPq of Brazil.

\section{Appendix}

The nonrelativistic reduction of the one pion exchange interaction is given by [29]

$$
\begin{aligned}
V^{O P E P} & =\frac{g_{A}^{2}}{4 \pi}\left(\frac{m_{\pi}}{2 f_{\pi}}\right)^{2}\left(\vec{\tau}_{1} \cdot \vec{\tau}_{2}\right)\left[\frac{1}{3}\left(\vec{\sigma}_{1} \cdot \vec{\sigma}_{2}\right)+\left(\frac{1}{\left(m_{\pi} r\right)^{2}}+\frac{1}{m_{\pi} r}+\frac{1}{3}\right) S_{12}\right] \frac{e^{-m_{\pi} r}}{r} \\
& =\left(\vec{\tau}_{1} \cdot \vec{\tau}_{2}\right)\left[V_{S S}^{O P E P}\left(\vec{\sigma}_{1} \cdot \vec{\sigma}_{2}\right)+V_{T}^{O P E P} S_{12}\right]
\end{aligned}
$$

The derivatives of the potentials with respect to the square of the pion mass can be simply stated as

$$
m_{\pi}^{2} \frac{\partial V_{S S}^{O P E P}}{\partial m_{\pi}^{2}}=\frac{1}{12 \pi}\left(\frac{m_{\pi}}{2 f_{\pi}}\right)^{2}\left\{2 g_{A} \frac{\partial g_{A}}{\partial m_{\pi}^{2}} m_{\pi}^{2}+g_{A}^{2}\left(1-\frac{m_{\pi} r}{2}\right)\right\} \frac{e^{-m_{\pi} r}}{r}
$$

and

$$
m_{\pi}^{2} \frac{\partial V_{T}^{O P E P}}{\partial m_{\pi}^{2}}=\frac{1}{4 \pi}\left(\frac{m_{\pi}}{2 f_{\pi}}\right)^{2}\left\{2 g_{A} \frac{\partial g_{A}}{\partial m_{\pi}^{2}} m_{\pi}^{2}\left(\frac{1}{\left(m_{\pi} r\right)^{2}}+\frac{1}{m_{\pi} r}+\frac{1}{3}\right)-\frac{g_{A}^{2}}{6}\left(1+m_{\pi} r\right)\right\} \frac{e^{-m_{\pi} r}}{r}
$$




\section{FIGURES}

FIG. 1. Derivative of the central component of the NN interaction, $m_{q} \frac{\partial V_{C}}{\partial m_{q}}$ as a function of the nucleon-nucleon relative distance in the Skyrme model. Solid curve corresponds to $m_{q} \frac{\partial V_{C}}{\partial m_{q}}$ with $f_{\pi}=93 \mathrm{MeV}$ and $e=4.0$. Dotted curve represents $V_{C}^{\left(m_{\pi}=138\right)}-V_{C}^{\left(m_{\pi}=0\right)}$.

FIG. 2. Derivative of the tensor component of the NN interaction, $m_{q} \frac{\partial V_{T}}{\partial m_{q}}$ as a function of the nucleon-nucleon relative distance in the Skyrme model. Solid curve corresponds to $m_{q} \frac{\partial V_{T}}{\partial m_{q}}$ with $f_{\pi}=93 \mathrm{MeV}$ and $e=4.0$. The dashed curve corresponds to the OPEP results. Dotted curve represents $V_{T}^{\left(m_{\pi}=138\right)}-V_{T}^{\left(m_{\pi}=0\right)}$.

FIG. 3. Derivative of the spin-spin component of the NN interaction, $m_{q} \frac{\partial V_{S S}}{\partial m_{q}}$ as a function of the nucleon-nucleon relative distance in the Skyrme model. The labels of the curves are the same as in Fig.2. 


\section{TABLES}

TABLE I. Nucleon observables $(O)$ and the corresponding derivatives with respect to the quark mass.

\begin{tabular}{|c|c|c||c|c|}
\hline \hline & \multicolumn{2}{|c||}{$f_{\pi}=54 \mathrm{MeV} e=4.84$} & \multicolumn{2}{c|}{$f_{\pi}=93 \mathrm{MeV} e=4.0$} \\
\hline & $O$ & $m_{q} \frac{\partial O}{\partial m_{q}}$ & $O$ & $m_{q} \frac{\partial O}{\partial m_{q}}$ \\
\hline$M_{N}$ & $938 \mathrm{MeV}$ & $52.2 \mathrm{MeV}$ & $1818 \mathrm{MeV}$ & $59.6 \mathrm{MeV}$ \\
\hline$g_{A}$ & 0.65 & -0.064 & 1.01 & -0.069 \\
\hline$g_{\pi N N}$ & 11.88 & -0.022 & 20.35 & -0.33 \\
\hline \hline
\end{tabular}

TABLE II. Contributions of the derivative of the NN potential and average kinetic energy to the nuclear sigma term. Calculations performed for the Skyrme Model with $f_{\pi}=93 \mathrm{MeV}$ and $\mathrm{e}=4.0$. The upper label in the nucleus symbol denotes the parametrization of the densities according to de Jager, de Vries and de Vries[21].

\begin{tabular}{|c|c|c|c|c|c|}
\hline \hline & $\bar{E}_{K}(\mathrm{MeV})$ & $\Sigma_{A}^{K}(\mathrm{MeV})$ & $\Sigma_{A}^{V}(\mathrm{MeV})$ & $\Sigma_{A}^{V+K}(\mathrm{MeV})$ & $\Sigma_{A}^{V+K} / A(\mathrm{MeV})$ \\
\hline${ }^{2} \mathrm{H}$ & 17.8 & -0.86 & -3.60 & -4.46 & -2.23 \\
\hline${ }^{3} \mathrm{H}$ & 46.1 & -2.3 & $\approx-14$ & $\approx-16$ & $\approx-5.4$ \\
\hline${ }^{3} \mathrm{He}$ & 46.1 & -2.3 & $\approx-14$ & $\approx-16$ & $\approx-5.4$ \\
\hline${ }^{4} \mathrm{He}^{(3 \mathrm{pF})}$ & 106 & -5.09 & -34.5 & -39.6 & -9.9 \\
\hline${ }^{10} \mathrm{~B}^{(\mathrm{h} . \mathrm{o} .)}$ & 228 & -10.9 & -107 & -118 & -11.8 \\
\hline${ }^{12} \mathrm{C}^{(3 \mathrm{pF})}$ & 274 & -13.1 & -157 & -170 & -14.2 \\
\hline${ }^{14} \mathrm{~N}^{(\mathrm{h} . \mathrm{O} .)}$ & 319 & -15.3 & -188 & -203 & -14.5 \\
\hline${ }^{16} \mathrm{O}^{(3 \mathrm{pF})}$ & 365 & -17.5 & -220 & -238 & -14.9 \\
\hline${ }^{20} \mathrm{Ne}^{(2 \mathrm{pF})}$ & 456 & -21.8 & -268 & -290 & -14.5 \\
\hline${ }^{24} \mathrm{Mg}^{(3 \mathrm{pF})}$ & 547 & -26.2 & -388 & -414 & -17.2 \\
\hline${ }^{32} \mathrm{~S}^{(3 \mathrm{pF})}$ & 730 & -35.0 & -557 & -592 & -18.5 \\
\hline${ }^{40} \mathrm{Ca}^{(3 \mathrm{pG})}$ & 912 & -43.8 & -727 & -771 & -19.3 \\
\hline${ }^{56} \mathrm{Fe}^{(3 \mathrm{pG})}$ & 1273 & -61.1 & -1144 & -1205 & -21.5 \\
\hline${ }^{118} \mathrm{Sn}^{(3 \mathrm{pG})}$ & 2690 & -129 & -2763 & -2892 & -24.5 \\
\hline${ }^{148} \mathrm{Sm}^{(2 \mathrm{pF})}$ & 3374 & -162 & -3556 & -3718 & -25.1 \\
\hline${ }^{197} \mathrm{Au}^{(2 \mathrm{pF})}$ & 4492 & -216 & -5187 & -5403 & -27.4 \\
\hline${ }^{208} \mathrm{~Pb}^{(3 \mathrm{pG})}$ & 4742 & -227 & -5274 & -5501 & -26.4 \\
\hline$\rho\left(0.17 \mathrm{fm}^{-3}\right)$ & $22.8 A$ & $-1.09 A$ & $-38.0 A$ & $-39.1 A$ & -39.1 \\
\hline \hline
\end{tabular}


TABLE III. Results for the isoscalar parameter of the optical potential including the potential sigma term contribution in Eq.(30). The ${ }^{12} \mathrm{C}$ and ${ }^{16} \mathrm{O}$ experimental values were estimated from the analysis described in Ref.[10] and for ${ }^{40} \mathrm{Ca}$ from the analysis of Ref.[11]. The value of $b_{0_{\text {eff }}}^{\text {free }}$ was obtained from the experimental value of the $\pi$-d scattering length [26] $\left(b_{0_{\mathrm{e} f f}}^{\text {free }}=-0.0099\left(m_{\pi}^{-1}\right)\right)$.

\begin{tabular}{|c|c|c|c|}
\hline \hline & $b_{0}^{V+K}$ & $b_{0}^{V+K}+b_{0_{\text {eff }}^{\text {free }}}$ & $b_{0_{\text {eff }}}^{(\exp )}$ \\
\hline${ }^{2} \mathrm{H}$ & -0.0025 & -0.0123 & $-0.0123(3)$ \\
\hline${ }^{3} \mathrm{H}$ & -0.0063 & -0.0162 & - \\
\hline${ }^{3} \mathrm{He}$ & -0.0063 & -0.0162 & -0.015 \\
\hline${ }^{4} \mathrm{He}^{(3 \mathrm{pF})}$ & -0.0110 & -0.0209 & $-0.0212(5)$ \\
\hline${ }^{10} \mathrm{~B}^{(\mathrm{h} . \mathrm{o})}$ & -0.0131 & -0.0230 & - \\
\hline${ }^{12} \mathrm{C}^{(3 \mathrm{pF})}$ & -0.0157 & -0.0256 & -0.0260 \\
\hline${ }^{14} \mathrm{~N}^{(\mathrm{h} . \mathrm{o} .)}$ & -0.0160 & -0.0259 & - \\
\hline${ }^{16} \mathrm{O}^{(3 \mathrm{pF})}$ & -0.0165 & -0.0264 & $-0.026^{(50 \mathrm{MeV})} ;-0.027^{(29 \mathrm{MeV})}$ \\
\hline${ }^{20} \mathrm{Ne}^{(2 \mathrm{pF})}$ & -0.0160 & -0.0259 & - \\
\hline${ }^{24} \mathrm{Mg}^{(3 \mathrm{pF})}$ & -0.0190 & -0.0289 & - \\
\hline${ }^{32} \mathrm{~S}^{(3 \mathrm{pF})}$ & -0.0204 & -0.0303 & $-0.033^{(25 \mathrm{MeV})}$ \\
\hline${ }^{40} \mathrm{Ca}^{(3 \mathrm{pF})}$ & -0.0213 & -0.0312 & -0.0304 \\
\hline$\rho_{e}=0.17 \mathrm{fm}{ }^{-3} / 2$ & -0.0216 & -0.0315 & \\
\hline \hline
\end{tabular}




\section{REFERENCES}

[1] T.D.Cohen, R.J.Furnstahl, and D.K.Griegel, Phys.Rev. Lett. 67, 961(1991).

[2] T.D.Cohen, R.J.Furnstahl, and D.K.Griegel, Phys.Rev. C45, 1881(1992).

[3] M.Gell-Mann, R.Oakes, and B.Renner, Phys. Rev.175, 2195(1968).

[4] T.H.R.Skyrme, Proc.R.Soc. London A260, 127(1961); A262, 237(1961).

[5] T.H.R.Skyrme, Nucl.Phys. 31, 556(1962).

[6] G.S.Adkins, C.Nappi and E.Witten, Nucl.Phys. B228, 552(1983). G.S.Adkins and C.Nappi, Nucl.Phys. B233, 109(1984). G.Adkins, Chiral Solitons, ed.K.F.Liu, World Scientific, Singapore, 1987.

[7] A.Jackson, A.D.Jackson and V.Paskier, Nucl.Phys.A432, 567(1985).

[8] R.Vinh Mau, M.Lacombe, B.Loiseau, W.N.Cottingham and P.Lisboa, Phys.Lett. B150, 259(1985).

[9] T.S.Walhout and J.Wambach, Phys.Rev.Lett. 67, 314 (1991); Int.J. Mod.Phys. E 1, 665(1992) .

[10] R.Seki and K.Masutani, Phys.Rev.C 27, 2799(1983).

[11] K.Masutani and R.Seki, Phys.Rev.C 38, 867(1988).

[12] L.L.Salcedo, K.Holinde, E.Oset, C.Schütz, Phys.Lett. B353, 1(1995).

[13] J.Delorme, G.Chanfray and M. Ericson, Nucl. Phys. A603, 239(1996).

[14] T.P.Cheng and L.F.Li, Gauge Theory of Elementary Particle Physics, Oxford Univ. Press, Oxford, 1994, p.160-165.

[15] J. Gasser, H.Leutwyler and M.E. Sainio, Phys. Lett. B253, 252(1991).

[16] G.Q.Li and C.M. Ko, Phys. Lett. B338, 118(1994).

[17] A.Delfino, Jishnu Dey, Mira Dey, M.Malheiro, Phys.Lett. B 363, 17(1995); Z.Phys. C71, 507(1996).

[18] R.Brockman and W.Weise, Phys.Lett. B367, 40(1996).

[19] G.E.Brown and M.Rho, Phys.Rep.269, 333(1996); Nucl.Phys. A596, 503(1996).

[20] G.Holzwarth, G.Pari and B.K.Jennings, Nucl.Phys. A515, 665(1990).

[21] C.W. de Jager, H. de Vries, C. de Vries, Atomic Data and Nucl. Data Tables 14, 479(1974).

[22] T.Ericson and W.Weise, Pions and Nuclei, Clarendon Press, Oxford, 1988.

[23] R.V.Reid, Ann. Phys. 50, 411(1968).

[24] R. Schiavilla, V.R. Phandaripande and R.B. Wiringa, Nucl.Phys.A449, 219(1986).

[25] J.M.Eisenberg and D.Koltun, Theory of Meson Interactions with Nuclei, Wiley, 1980.

[26] D.Chatellard et al., Phys.Rev.Lett. 74, 4157(1995).

[27] D.Sigg et al., Phys.Rev.Lett. 75, 3245(1995).

[28] R.Koch, Nucl.Phys. A448, 107(1986).

[29] M.R.Robilotta, private communication; J.L. Ballot, M. Ericson and M.R.Robilotta (to be published).

[30] W.Kluge, Rep.Prog.Phys. 54, 1251(1991). 
FIGURE 1

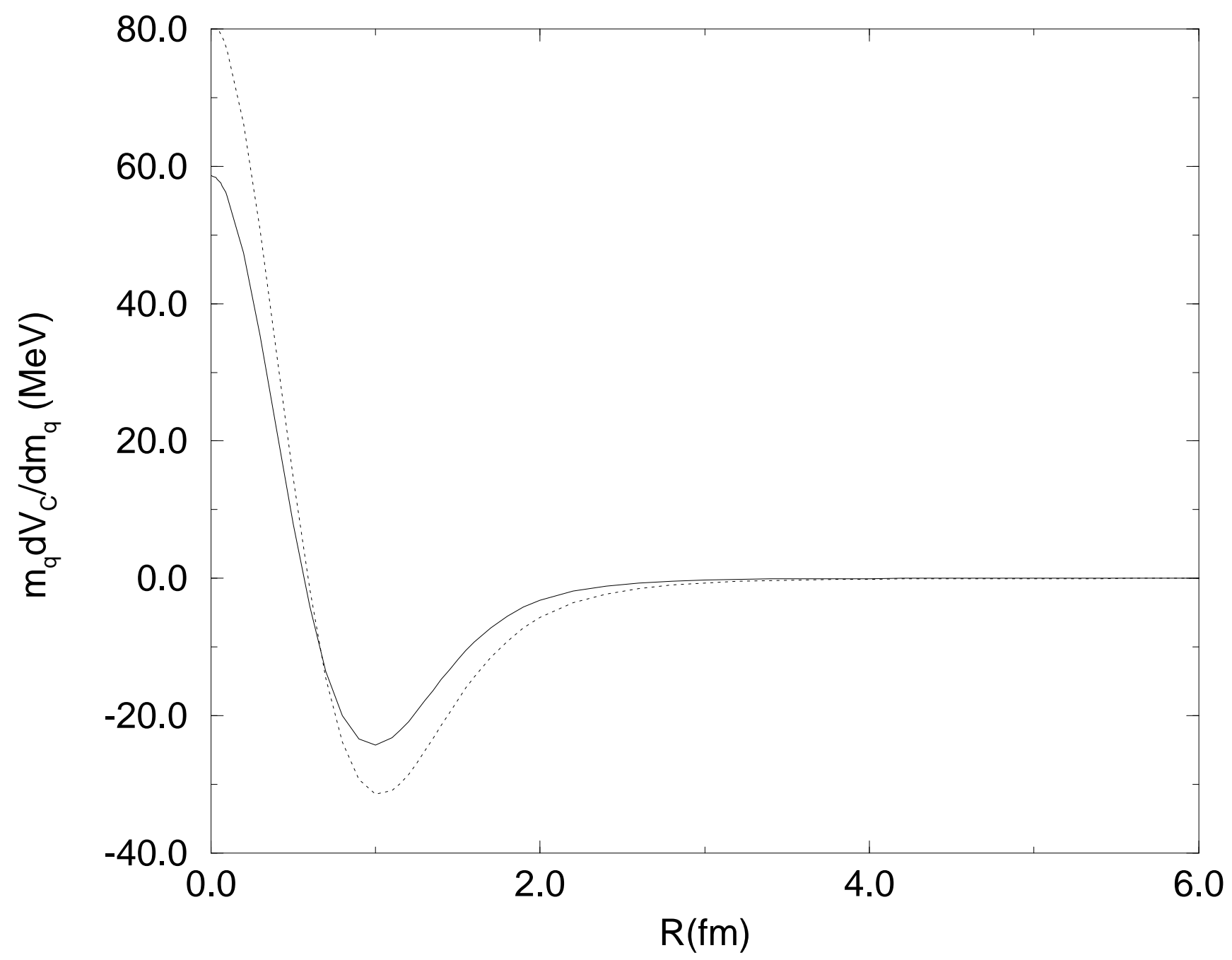


FIGURE 2

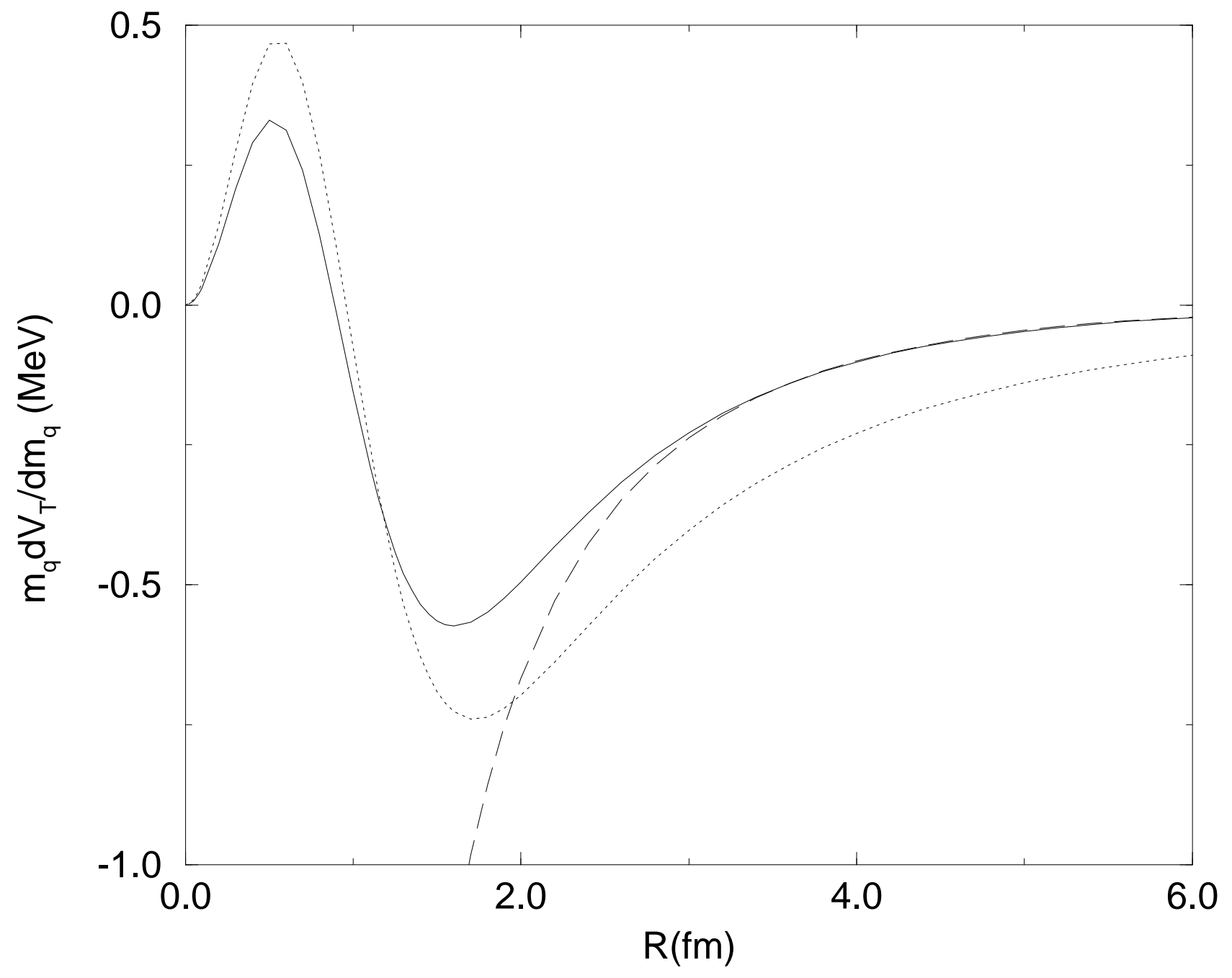


FIGURE 3

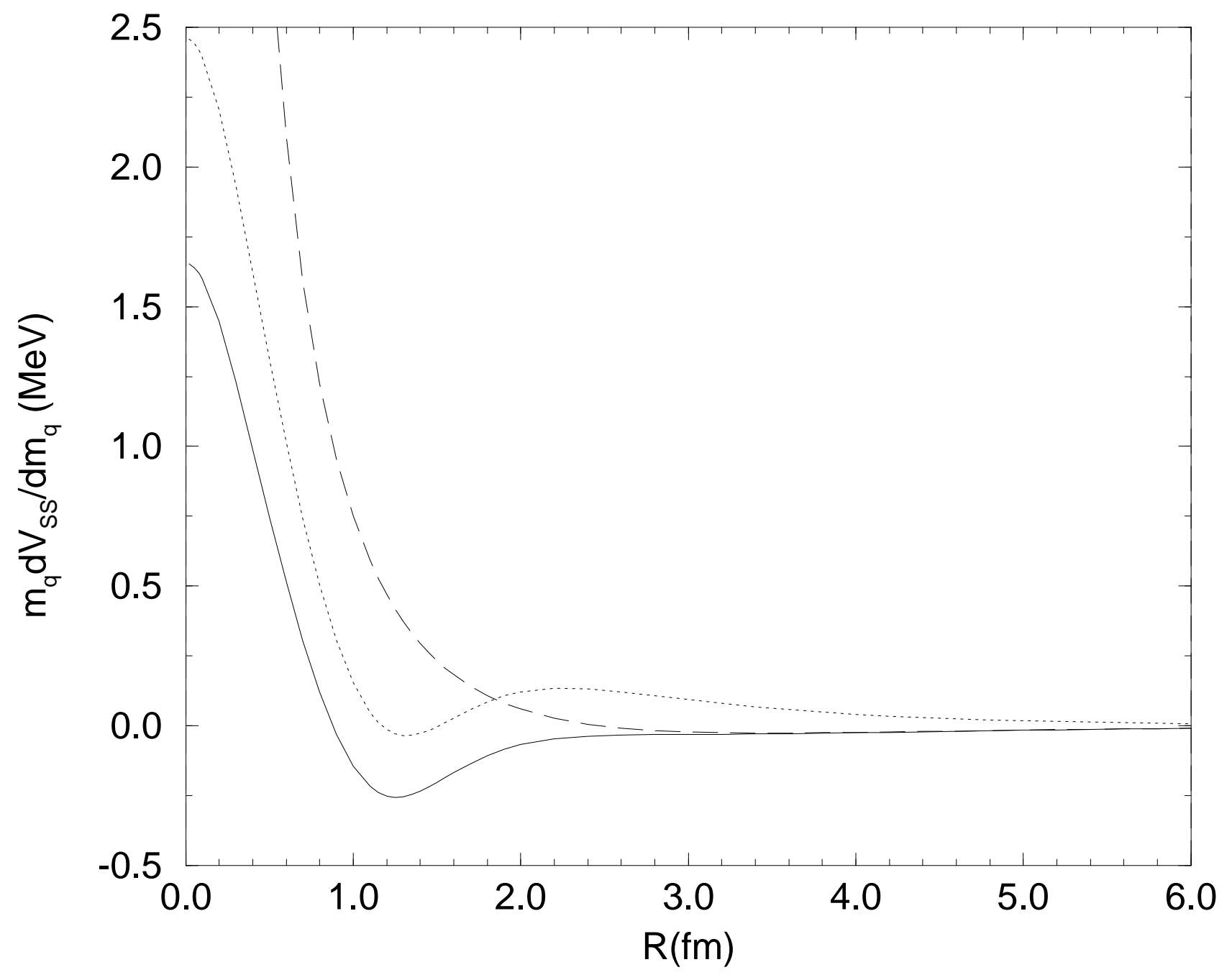

